\title{
Separation of Multiple Time Delays Using New Spectral Estimation Schemes
}

\author{
Mohammed A. Hasan, Mahmood R. Azimi-Sadjadi, Senior Member, IEEE, and Gerald J. Dobeck
}

\begin{abstract}
The problem of estimating multiple time delays in presence of colored noise is considered in this paper. This problem is first converted to a high-resolution frequency estimation problem. Then, the sample lagged covariance matrices of the resulting signal are computed and studied in terms of their eigenstructure. These matrices are shown to be as effective in extracting bases for the signal and noise subspaces as the standard autocorrelation matrix, which is normally used in MUSIC and the pencil-based methods. Frequency estimators are then derived using these subspaces. The effectiveness of the method is demonstrated on two examples: a standard frequency estimation problem in presence of colored noise and a real-world problem that involves separation of multiple specular components from the acoustic backscattered from an underwater target.
\end{abstract}

Index Terms - Data decimation, spectral estimation, underwater acoustics.

\section{INTRODUCTION}

$\mathbf{I}$ N UNDERWATER target detection using sonar, the presence of the targets can be verified by identifying certain clues about the target in the backscattered signal [1]-[3]. The first step in this process is to separate multiple specular returns from the backscattered signal so that the residual part can be analyzed more efficiently. Accurate separation can only be made possible if the time delays and amplitudes of multiple specular returns can accurately be estimated. Depending on the target geometry, beam width, and surrounding environment, the backscatter may contain several closely spaced specular returns with different amplitudes. This makes accurate separation of these components a very difficult task.

The problem of estimating time delays in the presence of colored noise arises in many different fields such as radar, sonar, seismic, and biomedical applications. The problem is typically transformed into a harmonic retrieval problem using Fourier transform. In recent years, there has been an increasing interest in model-based sinusoidal estimation. These models normally convert the nonlinear problem of estimating the frequencies into a simpler problem of estimating the parameters of a linear model. The desired information can

Manuscript received August 6, 1996; revised December 5, 1997. This work was supported by the Office of Naval Research (ONR 321TS). The Technical Agent was Coastal Systems Station, Panama City, FL. The associate editor coordinating the review of this paper and approving it for publication was Dr. Eric Moulines.

M. A. Hasan and M. R. Azimi-Sadjadi are with the Department of Electrical Engineering, Colorado State University, Fort Collins, CO 80523 USA (e-mail: azimi@engr.colostate.edu).

G. J. Dobeck is with the Coastal Systems Station, NSWC/DD, Panama City, FL 32407 USA.

Publisher Item Identifier S 1053-587X(98)03919-1. then be extracted from the estimated model parameters. The reliability of the first step depends on the accuracy of the estimation procedure, and that of the second depends on the sensitivity of the frequencies to the model parameters.

Among the well-known approaches to this problem are Prony's method and the maximum likelihood method [4], [5]. Prony's method and its variants are based on the assumption that a pure sum of sinusoids fits an autoregressive (AR) model whose parameters can be determined from a finite number of data points. In the presence of measurement noise, the accuracy of Prony-type methods deteriorates rapidly. To overcome this sensitivity problem, many alternative schemes were proposed. Eigenvalue and singular value-based methods such as that proposed by Tufts and Kumaresan [5] perform well for moderate signal-to-noise-ratio (SNR) cases. In most of these methods, the autocorrelation of a data matrix is computed, and signal and noise subspaces are then determined using the eigenvalues and the eigenvectors of this matrix. First, the number of sources is determined from the number of significant eigenvalues. The corresponding eigenvectors then form the signal subspace, while those corresponding to the minimal eigenvalues are chosen as a basis of the noise subspace. The MUSIC method [6] is a procedure that utilizes the noise subspace eigenvectors.

Generally, modern high-resolution subspace estimation schemes are of three types.

1) extrema searching techniques like spectral MUSIC [6];

2) polynomial rooting techniques such as Root-MUSIC [7] and Pisarenko methods [4];

3) matrix shifting methods such as ESPRIT [8] and matrix pencil methods [9].

The statistical efficiency of these methods are studied in [10] and [11]. However, these methods are computationally demanding since they involve the computation of each singular eigenvector and corresponding eigenvalue. To reduce the computational cost associated with these subspace methods, various alternatives were proposed [12]-[14].

More recently, Nagesha and Kay [15] have developed a maximum likelihood (ML)-based method in which the color noise is modeled by an AR process with unknown parameters. This method uses canonical factorization of density function and the application of nonlinear parameter transformation in order to reduce the problem into maximization of a concentrated likelihood function that is carried out numerically. In [16], Friedlander and Francos derived a closed form exact Cramér-Rao bound (CRB) on the achievable accuracy for joint estimation of unknown harmonics as the AR parameters 
of the colored noise. They also showed how this bound can be computed from the conditional likelihood function of the observations.

The goal of this paper is to develop new high-resolution spectral estimation schemes to accurately estimate the time delays associated with multiple specular components in the acoustic backscattered signal from underwater targets. It is shown that all the lagged data correlation matrices contained approximately the same information about the noise and signal subspaces. This property is used to develop several new algorithms using high-lag correlation matrices. These methods, which can be viewed as extensions to the MUSIC and Pencilbased methods [6], [7], [9], are primarily used for the purpose of signal-noise subspace decomposition in situations where the noise is colored.

This paper is organized as follows. Section II presents formulation of the time delay estimation problem as a sinusoidal frequency estimation. The MUSIC frequency estimation scheme is then briefly reviewed. Based on correlation matrices with lags, two new algorithms are developed in Sections III and IV. Enhanced resolution using the decimation process and correlations of higher order lags are presented in Section V. Finally, simulation results on two different examples are provided in Section VI.

\section{PRoblem Formulation AND FreQuency Estimation}

Let us consider the acoustic backscattered signal $z(t)$ in the form of

$$
z(t)=\sum_{i=1}^{M} h_{i}(t) * x\left(t-\Delta_{i}\right)+u(t)
$$

where the first term on the right-hand side represents the effects of target specular returns and volume and surface reverberation, and the second term $u(t)$ accounts for the effects of additive ambient noise. In the first term, $h_{i}(t)$, $i \in[1, M]$ represents effects of media or reverberation, $x(t)$ is the transmitted or incident signal, $\Delta_{i}$ is the unknown delay associated with the $i$ th component, and $*$ is the symbol for convolution operation. Reverberation results from random distribution of acoustic scatterers due to inhomogeneities and roughness at the top and bottom surfaces of the ocean (surface reverberation) as well as those corresponding to biological sources in the water column (volume reverberation). The effects of media and reverberation can be modeled [3] by an unknown constant gain $A_{i}$.

Assuming bandlimited signals, (1) can be transformed into a sinusoidal frequency estimation problem by taking the Fourier transform of (1), dividing by $X(w)$, and sampling the frequency axis to yield

$$
y(k)=\sum_{i=1}^{M} A_{i} e^{-j\left(2 \pi k f_{i}-\phi_{i}\right)}+v(k), \quad k \in[1, N]
$$

where $f_{i}:=\Delta_{i} / N$. Note that as in [6], the phase $\phi_{i}$, which is assumed to be an i.i.d. random variable uniformly distributed over $[0,2 \pi)$, is included for the sake of generality. Now, the problem is reduced to estimating the optimum number of sinusoids $M$, their magnitudes $A_{i}$, and their frequencies $f_{i}$ using the noisy data $y(k), k=1,2, \cdots, N$.

Eigenstructure methods [4]-[6] are among the most wellknown high-resolution sinusoidal estimation methods available in the literature. The main objective of these methods is to generate orthogonal eigenspaces consisting of the signal and noise subspaces from the estimated autocorrelation matrix. Frequency estimators such as MUSIC [6] can then be applied to provide estimates of the unknown frequencies. Here, we briefly review the conventional MUSIC method for frequency estimation in presence of white noise.

If $y(k)$ and $v(k)$ are partitioned into overlapping blocks of size $L$, then (2) can be rewritten in vector form as

$$
\mathbf{y}_{k}:=\sum_{i=1}^{M} A_{i} e^{j \phi_{i}} \mathbf{e}\left(k, f_{i}\right)+\mathbf{n}_{k}
$$

where $\mathbf{y}_{k}:=\left[\begin{array}{llll}y(k) & y(k+1) & \cdots & y(k+L-1)\end{array}\right]^{T}$, $\mathbf{n}_{k}:=\left[\begin{array}{llll}v(k) & v(k+1) & \cdots & v(k+L-1)\end{array}\right]^{T}$, and $\mathbf{e}(k, f):=\left[\begin{array}{llll}e^{-j 2 \pi k f} & e^{-j 2 \pi(k+1) f} & \cdots & e^{-j 2 \pi(k+L-1) f}\end{array}\right]^{T}$, $T$ denotes matrix transposition and $L \geq M$. The vector $\mathbf{e}(k, f)$ can be expressed as $\mathbf{e}(k, f)=e^{-j 2 \pi k f} \mathbf{e}(f)$, where $\mathbf{e}(f):=\left[1 e^{-j 2 \pi f} e^{-j 2 \pi 2 f} \cdots e^{-j 2 \pi(L-1) f}\right]^{T}$. The vectors $\left\{\mathbf{e}\left(f_{i}\right)\right\}_{i=1}^{M}$ are sometimes referred to as the signal vectors.

As shown in [6], the frequencies $f_{i}$ can be estimated by decomposing the autocorrelation matrix $R_{y}=E\left[\mathbf{y}_{k} \mathbf{y}_{k}^{*}\right]$ into the signal and noise subspaces. Note that here, sample averaged statistics are used as approximations to the ensemble averaged statistics since the number of blocks is usually very large. Now, assuming that the eigenvalues $\left\{\lambda_{i}\right\}_{i=1}^{L}$ of $R_{y}$ are sorted in decreasing order so that $\lambda_{1} \geq \lambda_{2} \geq \cdots \geq \lambda_{L}$, with corresponding orthogonal eigenvectors $v_{1}, v_{2}, \cdots, v_{L}$, respectively, if the noise $v(k)$ is white with variance $\sigma_{v}^{2}$ and $L>M$, then there exists an $M$ such that $\lambda_{1} \geq \lambda_{2} \geq \cdots \geq$ $\lambda_{M} \gg \lambda_{M+1}=\cdots=\lambda_{L}=\sigma_{v}^{2}$. The ranges of the matrices $V_{1}=\left[\begin{array}{llll}v_{1} & v_{2} & \cdots & v_{M}\end{array}\right]$ and $V_{2}=\left[\begin{array}{llll}v_{M+1} & v_{M+2} & \cdots & v_{L}\end{array}\right]$ are called the signal and noise subspaces, respectively. In the noiseless case, the column space of $V_{1}$ is the signal subspace of the signal vectors $\mathbf{e}\left(f_{1}\right), \cdots, \mathbf{e}\left(f_{M}\right)$. Practically, by examining the eigenvalues of $R_{y}$, we get an estimate for the $M$ most significant eigenvalues, in which case, $M$ represents the number of sinusoids contained in the signal part. Once this decomposition is done, using, usually, the singular value decomposition (SVD) algorithm [17], the MUSIC frequency estimator [6] can be applied as

$$
P(f)=\frac{1}{\mathbf{e}^{*}(f) V_{2} V_{2}^{*} \mathbf{e}(f)}=\frac{1}{L-\mathbf{e}^{*}(f) V_{1} V_{1}^{*} \mathbf{e}(f)}
$$

The estimate of the frequencies are generated by plotting $P(f)$ and identifying the peaks associated with the locations of the $f_{i}$ 's. Once $f_{i}$ 's are estimated, the amplitudes $A_{i}$ can be obtained using a least squares (LS)-based estimator.

In the sequel, we will show how correlations with lags can be used to develop new approaches for the estimation of the parameters of complex sinusoids corrupted with colored noise. 


\section{GENERALIZED EIGENSTRUCTURE OF LAGGED AUTOCORRELATION MATRICES}

In this section, we investigate the eigenstructure of lagged correlation matrices of sinusoids and use some of their properties to extend the eigendecomposition-based methods so that correlations with lags can be used to extract the signal and noise subspaces. The idea is that for a wide range of random processes, the higher the lag, the less noisier the data correlations are since the noise becomes more decorrelated at higher lags. To understand the eigenstructure of lagged autocorrelation matrices of sinusoids, assume that $y(k)=$ $x(k)+v(k)$, where $x(k)=\sum_{i=1}^{M} A_{i} e^{-2 \pi j k\left(f_{i}-\phi_{i}\right)}$, and $v(k)$ is a random process modeled by an $s$ th-order moving average (MA) process $v(k)=a_{0} w(k)+a_{1} w(k-1)+\cdots+a_{s} w(k-s)$, where $w(k)$ is a zero mean white noise with variance $\sigma_{w}^{2}$. This idea is similar to those in [15] and [16]. Let $r_{v}(m)$ be the autocorrelation function of $v(k)$ defined by $r_{v}(m)=$ $E\left[v(k) v^{*}(k-m)\right]$. It can easily be verified that

$$
r_{v}(m)= \begin{cases}\sigma_{w}^{2} \sum_{i=0}^{s} a_{i} a_{i-m}^{*} & \text { for } m \in[0, s] \\ r_{v}(m)=0 & \text { for } m>s\end{cases}
$$

holds for the autocorrelation of $v(k)$. Further, we have $r_{v}(-m)=r_{v}^{*}(m)$ for all $m$. The white process is obviously the simplest case in which $r_{v}(m)=0$ holds for $|m| \geq 1$. This implies that for this case, the new sequence $\left\{r_{y}(m)\right\}_{m=s+1}^{\infty}$ would contain fewer noise effects than the original one. This observation will be used to develop algorithms for estimating the $f_{i}$ 's.

Proposition 3.1: Let $y(k)=x(k)+v(k)$, with $x(k)=$ $\sum_{i=1}^{M} A_{i} e^{-j\left(2 \pi k f_{i}-\phi_{i}\right)}$, and $x(k)$ and $v(k)$ are zero mean and independent process; then, $r_{y}(m)=r_{x}(m)+r_{v}(m)$, where

$$
r_{x}(m)=\sum_{i=1}^{M} A_{i} A_{i}^{*} e^{-2 \pi j m f_{i}}
$$

and $r_{y}(m)=E\left[y(k) y^{*}(k-m)\right]$.

Proof: This result follows directly using $E\left[e^{j\left(\phi_{i}-\phi_{j}\right)}\right]=$ $\delta(i-j)$.

The last result implies that for $m>s, r_{y}(m)$ is a sum of pure sinusoids but with squared amplitudes. Thus, like the original signal, the sequence $r_{y}(m), m=0,1,2, \cdots, N$ is formed of a sum of sinusoids and the autocorrelation of the noise $r_{v}(m)$, which becomes zero for $m>s$. Consequently, the estimates of the frequencies and amplitudes can be done more efficiently using $r_{y}(m)$. In addition, the effect of phase does not appear in this domain, compared with the original observation domain. This significantly simplifies the computation of the amplitudes $A_{i}^{\prime} s$. The next proposition reveals some of the useful properties of the auto-correlation matrix $R_{x}(m)$ defined as $R_{x}(m)=E\left[\mathbf{x}_{k} \mathbf{x}_{k-m}^{*}\right]$.

Proposition 3.2: Assume that $x(k)$ as in Proposition 3.1, $A_{i} \neq 0$ for $i=1, \cdots, M$, and $L \geq M$, Then, we have (6), as shown at the bottom of the page. Moreover, the following relations hold.

i) $\quad R_{x}(m)=F R_{x}(m) F^{*}$

ii) $R_{x}(m)=F^{r} R_{x}(m) F^{* r}$.

iii) $R_{x}(m)=F R_{x}(m-1)=R_{x}(m+1) F^{*}$.

iv) $R_{x}(m)=F^{m} R_{x}(0)=R_{x}(2 m) F^{* m}$.

v) $R_{x}(m+r)=F^{r} R_{x}(m)=R_{x}(m+2 r) F^{* r}$.

vi) For each integer $m, R_{x}(m)$ is of rank $M$.

Here, $F$ is the companion matrix of the polynomial whose roots hold the unknown frequencies

$$
\Delta(z)=z^{L-M} p(z)=z^{L}+c_{1} z^{L-1}+\cdots+c_{L}=0
$$

where $p(z)=\prod_{i=1}^{M}\left(z-e^{-2 \pi j f_{i}}\right)$, and

$$
F=\left[\begin{array}{cccccc}
0 & 1 & 0 & \cdots & 0 & 0 \\
0 & 0 & 1 & \cdots & 0 & 0 \\
\vdots & \vdots & \vdots & \vdots & \vdots & \vdots \\
0 & 0 & 0 & \cdots & 0 & 1 \\
-c_{L} & -c_{L-1} & -c_{L-2} & \cdots & -c_{2} & -c_{1}
\end{array}\right] .
$$

Proof: See Appendix A.

Remark 3.1: The approximate equality $R_{y}(m) \approx R_{x}(m)$, when $m$ is large, ensures the existence of eigenvalues of matrix $R_{y}(m)$, which are close to zero. This approximation holds well for a large class of stationary noise processes for which $r_{v}(m) \rightarrow 0$ as $m \rightarrow \infty$. It must be noticed that when $L>M$, matrix $F^{*}$ is singular with one-dimensional (1-D) null space spanned by the vector $\left[\begin{array}{lllllll}0 & 0 & \cdots & c_{M} & c_{M-1} & \cdots & 1\end{array}\right]^{T}$. Of course, the ultimate goal is to determine the $c_{i}$ 's from the noisy data $y(k)$ or the correlation sequence $r_{y}(k)$ by gaining eigenstructure information concerning $F$. Since $r_{y}(m)=$ $r_{x}(m)$ for $m>s$, the sequence $\left\{r_{y}(m)\right\}_{m=s+1}^{N}$ can ideally be used to determine the $c_{i}$ 's.

Remark 3.2: Postmultiplying both sides of v) of Proposition 3.2 by $W$, where $W$ is any orthogonal matrix such that

$$
R_{x}(m)=\left[\begin{array}{cccc}
\sum_{i=1}^{M} A_{i} A_{i}^{*} e^{-2 \pi j m f_{i}} & \sum_{i=1}^{M} A_{i} A_{i}^{*} e^{-2 \pi j(m-1) f_{i}} & \cdots & \sum_{i=1}^{M} A_{i} A_{i}^{*} e^{-2 \pi j(m-L+1) f_{i}} \\
\sum_{i=1}^{M} A_{i} A_{i}^{*} e^{-2 \pi j(m+1) f_{i}} & \sum_{i=1}^{M} A_{i} A_{i}^{*} e^{-2 \pi j m f_{i}} & \cdots & \sum_{i=1}^{M} A_{i} A_{i}^{*} e^{-2 \pi j(m-L+2) f_{i}} \\
\vdots & \vdots & \vdots & \vdots \\
\sum_{i=1}^{M} A_{i} A_{i}^{*} e^{-2 \pi j(m+L-1) f_{i}} & \sum_{i=1}^{M} A_{i} A_{i}^{*} e^{-2 \pi j(m+L-2) f_{i}} & \cdots & \sum_{i=1}^{M} A_{i} A_{i}^{*} e^{-2 \pi j m f_{i}}
\end{array}\right] .
$$


$F^{*} W=W S$ and $S$ is upper triangular with eigenvalues $\left\{e^{-2 \pi j f_{1}}, \cdots, e^{-2 \pi j f_{M}}, 0, \cdots, 0\right\}$, we obtain $R_{x}(m) W=$ $R_{x}(m+r) W S^{r}$. Hence, $R_{x}(m)=R_{x}(m+r) W S^{r} W^{*}$. Note that matrices $\left\{U_{r}=W S^{r} W^{*}\right\}_{r>0}$ are independent of $m$. This provides a flexibility in choosing $m$ since $R_{y}(m)$ is less noisy for large $m$. This property will be used to develop an algorithm to recover the signal and noise subspaces, which are then used to estimate the frequencies.

Remark 3.3: Note that vi) of Proposition 3.2 implies that if the observed signal has no additive noise and $L \geq M$, then the rank of $R_{y}(m)$ is $M$ for each integer $m$. When noise is added to $x(k)$, the resulting autocorrelation data matrix $R_{y}(m)$ tends to have $M$ larger generalized eigenvalues associated with the signal subspace and $L-M$ smaller eigenvalues associated with the noise subspace. Thus, by examining the diagonal elements of $S$ in Remark 3.2, we can estimate $M$.

As indicated in vi) of the last result, when $L>M$, matrix $R_{x}(m)$ is singular. Its null space is determined next, where the notation $\mathcal{N}\left[R_{x}(m)\right]$ denotes the null space of $R_{x}(m)$.

Proposition 3.3: Assume that $L>M$; then, $R_{x}(m)$ is singular for each $m \geq 0$, and $\mathcal{N}\left[R_{x}(m)\right]=\mathcal{N}\left(F^{* L-M}\right)=$ span of columns of $\bar{G}$, where

$$
G=\left[\begin{array}{cccccccc}
c_{M} & c_{M-1} & \cdots & 1 & 0 & 0 & \cdots & 0 \\
0 & c_{M} & c_{M-1} & \cdots & 1 & 0 & \cdots & 0 \\
\vdots & \vdots & \vdots & \vdots & \vdots & \vdots & \vdots & \vdots \\
0 & \cdots & 0 & 0 & c_{M} & c_{M-1} & \cdots & 1
\end{array}\right]^{T}
$$

Additionally

$$
\mathcal{N}\left[R_{x}^{*}(m)\right]=\mathcal{N}\left[R_{x}(m)\right]=\text { column space of } G
$$

Proof: See Appendix B.

Note that the column space of $G$ and $G B$ are the same for any $M \times M$ nonsingular matrix $B$. For computational convenience, matrix $B$ is typically chosen so that $G B$ is orthogonal, as in the case of MUSIC [6]. The practical significance of Proposition 3.3 lies in the fact that it can be used to estimate the frequencies by estimating the coefficients $c_{j}$ of $\Delta(z)$ defined in Proposition 3.2. To see this, let $Z=$ $\left[1 z \cdots z^{L-1}\right]^{T}$, and $h(z)=Z^{*} G G^{* T} Z$; then

$$
\begin{aligned}
h(z)= & {\left[p\left(z^{*}\right), z^{*} p\left(z^{*}\right), \cdots, z^{* L-1} p\left(z^{*}\right)\right] } \\
& \cdot\left[p(z), z p(z), \cdots, z^{L-1} p(z)\right]^{T} \\
= & |p(z)|^{2}\left\{\sum_{i=0}^{L-1}|z|^{2 i}\right\}
\end{aligned}
$$

where $p(z)$ is defined in (7a). Practically, it is not numerically feasible to compute $G$; however, an orthogonal matrix that has the same column space can easily be estimated using the SVD decomposition. When $z$ is on the unit circle, then $h(z)=L|p(z)|^{2}$. This implies that $h(z)=0$ if and only if $p(z)=0$. In the MUSIC algorithm [6], $1 / h(z)$ is typically plotted for $z$ on the unit circle, and peaks are observed for their locations. In ROOT-MUSIC [7], the zeros of $h(z)$ are computed over the complex plane, and the frequencies are chosen among those close to the unit circle.

Remark 3.4: A nonzero eigenvector of $F$ has the form $Z_{i}=\left[\begin{array}{lllll}1 & z_{i} & \cdots & z_{i}^{L-1}\end{array}\right]^{T}$, where $z_{i}=e^{-2 \pi j f_{i}}, i \in[1, M]$ are the eigenvalues of $F$, i.e., matrix $V=\left[\begin{array}{llll}Z_{1} & Z_{2} & \cdots & Z_{M}\end{array}\right]$ diagonalizes $F$. Note that the frequency estimation problem reduces to finding the eigenvalues of $S$, which are the same as those of $F$ since $F$ and $S$ are similar (Remark 3.2).

Similar results as those of Proposition 3.2 can be obtained by defining a new matrix $\bar{R}_{x}(m, r)=R_{x}(m-r)+R_{x}^{*}(m+r)$. As can be observed from $(6), R_{x}(m)$ is Hermitian if and only if $m=0$. However, for numerical advantages, we consider the Hermitian matrix $\bar{R}_{x}(m, 0)=R_{x}(m)+R_{x}^{*}(m), m>0$, which has the following properties.

Corollary 3.4: Let $\bar{R}_{y}(m, r)$ and $F$ be as defined before; then, for each integer $r \geq 1$

i) $\quad \bar{R}_{x}(m, 0)=F \bar{R}_{x}(m, 1)=\bar{R}_{x}^{*}(m, 1) F^{*}$;

ii) $\bar{R}_{x}(m, 0)=F^{r} \bar{R}_{x}(m, r)=\bar{R}_{x}^{*}(m, r) F^{* r}$.

Proof: This follows from the observation that $R_{x}(m)+$ $R_{x}^{*}(m)=F R_{x}(m-1)+\left[R_{x}(m+1) F^{*}\right]^{*}=F R_{x}(m-1)+$ $F R_{x}^{*}(m+1)=F\left[R_{x}(m-1)+R_{x}^{*}(m+1)\right]$. Corollary 3.4ii) follows from iterating i).

Q.E.D.

Multiplying both sides if Corollary 3.4ii) by $W^{*}$, where $F^{*} W=W S$, yields

$$
\bar{R}_{x}(m, 0) W=\bar{R}_{x}^{*}(m, r) W S^{r}
$$

or equivalently

$$
\bar{R}_{x}(m, 0)=\bar{R}_{x}^{*}(m, r) U_{r}
$$

where $U_{r}=W S^{r} W^{*}$. As in the case of $R_{x}(m)$, matrix $U_{r}$ is independent of $m$.

This result, along with Proposition 3.2, shows that $R_{x}(m)$ and $\bar{R}_{x}(m, r)$, for $m=1,2, \cdots$, and $r=0,1, \cdots$, contain as much information about the signal and noise subspaces as the conventional correlation matrix $R_{x}(0)$ in that all of these can be utilized to determine $W$. The nonzero eigenvectors of the generalized eigenvalue problem (9) are the $Z_{i}$ 's defined above. Let $W$ in (9) be partitioned as $W=\left[W_{1} W_{2}\right]$ such that $W_{1}$ corresponds to the most significant $M$ eigenvalues of $S^{r}$, and $W_{2}$ corresponds to the least significant $L-M$ eigenvalues of $S^{r}$, respectively. Since $Z_{i}$ is in the signal subspace, $Z_{i}=$ $W_{1}\left(W_{1}^{*} W_{1}\right)^{-1} W_{1}^{*} Z_{i}$, and thus, $Z^{*} W_{1}\left(W_{1}^{*} W_{1}\right)^{-1} W_{1}^{*} Z=L$ if and only if $Z$ is in the signal subspace that is spanned by the $Z_{i}$ 's. Similarly, $Z_{i}^{*} W_{2}\left(W_{2}^{*} W_{2}\right)^{-1} W_{2}^{*} Z_{i}=0$ if and only if $Z$ is a linear combination of the $Z_{i}$ 's. By plotting $P_{1}(z)=1 /\left[L-Z^{*} W_{1}\left(W_{1}^{*} W_{1}\right)^{-1} W_{1}^{*} Z\right]$, the frequencies will be close to the locations of the peaks of $P_{1}(z)$. An algorithm that utilizes the ideas of Corollary 3.4 is presented next.

Algorithm 1:

i) Choose $m$ sufficiently large such that $m>s+L$, $r \geq 1$, and estimate the lagged sample correlations $R_{y}(m)$ and $R_{y}(m+r)$ of the data sequence $y(k)$ for $k=1, \cdots, N$.

ii) Solve the generalized eigenvalue problem $z \bar{R}_{y}(m, 0) w=\bar{R}_{y}(m, r) w$, and form the decomposition $\bar{R}_{y}(m+r) W=\bar{R}_{y}(m) W S^{r}$. 
iii) Let $W_{1}$ and $W_{2}$ be the columns of $W$ that correspond to the most significant $M$ eigenvalues of $S^{r}$ and the least significant $L-M$ eigenvalues of $S^{r}$, respectively.

iv) Let $P_{1}(f):=L-\mathbf{e}^{*}(f) W_{1}\left(W_{1}^{*} W_{1}\right)^{-1} W_{1}^{*} \mathbf{e}(f)=$ $\mathbf{e}^{*}(f) W_{2}\left(W_{2}^{*} W_{2}\right)^{-1} W_{2}^{*} \mathbf{e}(f)$. Then, the zeros of $P_{1}(f)$ are the estimated frequencies. Note that if $W_{1}$ has orthogonal columns, then $P_{1}(f)=$ $\mathbf{e}^{*}(f) W_{1} W_{1}^{*} \mathbf{e}(f)$. Orthogonal matrices can be automatically generated if the QZ algorithm [17], [18] is used.

\section{EIGENSTRUCTURE OF CORRELATIONS WITH HIGHER ORDER LAGS}

As mentioned in Section III, the main motivation behind using higher lags of correlations lies in the idea of suppressing the effects of colored noise. This leads to a more efficient and practical way of estimating the desired unknowns. In this section, we extend the eigendecomposition-based methods so that correlations with lags can be used to extract the signal and noise subspaces, as shown in the following result.

Proposition 4.1: There exist matrices $U=\left[\begin{array}{ll}U_{1} & U_{2}\end{array}\right]$ and upper triangular matrix $S$ such that $R_{x}(m) U=U H_{m}$, where $U_{1}=V B_{1}$ and $U_{2}=G B_{2}$, and $H_{m}=\left[\begin{array}{cc}S^{m} & 0 \\ 0 & 0\end{array}\right]$, where $V=\left[\begin{array}{lll}Z_{1} & \cdots & Z_{M}\end{array}\right]$ and $G$ are as defined in (8), and $B_{1}$ and $B_{2}$ are nonsingular matrices, i.e., $U_{1}$ and $U_{2}$ span the signal and noise subspaces of $R_{x}(m)$, respectively.

Proof: It is shown in Appendix A that $R_{x}(m)=$ $V D S^{m} D^{*} V^{*}$ and $R_{x}(m) G=0$, and it follows that

$$
\begin{aligned}
R_{x}(m)\left[\begin{array}{ll}
V & G
\end{array}\right] & =\left[\begin{array}{ll}
V D S^{m} D^{*} V^{*} V & 0
\end{array}\right] \\
& =\left[\begin{array}{ll}
V & G
\end{array}\right]\left[\begin{array}{cc}
D S^{m} D^{*} V^{*} V & 0 \\
0 & 0
\end{array}\right] .
\end{aligned}
$$

Let $Q$ be a nonsingular matrix that upper triangulizes $D S^{m} D^{*} V^{*} V$, i.e., $Q^{-1} D S^{m} D^{*} V^{*} V Q$ is upper triangular; then

$$
R_{x}(m)\left[\begin{array}{ll}
V Q & G
\end{array}\right]=\left[\begin{array}{ll}
V Q & G
\end{array}\right]\left[\begin{array}{cc}
H_{m} & 0 \\
0 & 0
\end{array}\right]
$$

where $H_{m}=Q^{-1} D S^{m} D^{*} V^{*} V Q$ is upper triangular. The conclusion follows by setting $U_{1}=V Q$ and $U_{2}=$ $G B_{2}$.

Q.E.D.

The practical value of Proposition 4.1 is that the eigenvalues of $R_{x}(m)$ are the same for all $m$ and that the signal subspace is spanned by the columns of $U_{1}=V B_{1}$ for some nonsingular matrix $B_{1}$, and the noise subspace is spanned by the null space given by the columns for $G$, i.e., the null space is generated by $U_{2}=G B_{2}$ for any $L-M \times L-M$ nonsingular matrix $B_{2}$. In the presence of noise, the $M$ most significant eigenvectors of $R_{y}(m)$ span a perturbed version of the signal subspace, whereas the least $L-M$ significant eigenvectors span a perturbed version of the noise subspace. This observation can be utilized to develop an algorithm that can be considered to be an extension of MUSIC. Owing to numerical robustness reasons, the Hermitian matrix $\left[R_{y}(m)+R_{y}^{*}(m)\right] / 2$ is used in the next algorithm to determine the signal and noise subspaces that are the same for all $m$. In particular, $\mathcal{N}\left[R_{x}(m)+\right.$
$\left.R_{x}^{*}(m)\right]=\mathcal{N}\left[R_{x}(m)\right]=\mathcal{N}\left[R_{x}^{*}(m)\right]$. An algorithm that utilizes this idea is presented next.

Algorithm 2:

i) Estimate the sample correlation matrix of the data sequence $y(k)$ for $k=1, \cdots, N$.

ii) Compute the eigenstructure of the Hermitian matrix $R_{y}(m)+R_{y}^{*}(m)$ so that $R_{y}(m)+R_{y}^{*}(m)=U \Sigma U^{*}=$ $\left[\begin{array}{ll}U_{1} & U_{2}\end{array}\right]\left[\begin{array}{cc}\Sigma_{1 y} & 0 \\ 0 & \Sigma_{2 y}\end{array}\right]\left[\begin{array}{ll}U_{1} & U_{2}\end{array}\right]^{*}$, where $U_{1}$ and $U_{2}$ are orthogonal whose columns span the signal and the noise subspaces, respectively. Here, $\Sigma_{1 y}$ holds the $M$ principal most significant eigenvalues, and $\Sigma_{2 y}$ holds the $L-M$ nonprincipal least significant eigenvalues of $R_{y}(m)$.

iii) Let $P_{2}(f)=\mathbf{e}^{*}(f) U_{2}\left(U_{2}^{*} U_{2}\right)^{-1} U_{2}^{*} \mathbf{e}(f)=L-$ $\mathbf{e}^{*}(f) U_{1}\left(U_{1}^{*} U_{1}\right)^{-1} U_{1}^{*} \mathbf{e}(f)$ and plot $1 / P_{2}(f)$. The peaks indicate the locations of the $f_{i}$ 's.

Remark 4.1-Choices of $L$ and $M$ : As shown previously, in the noise-free case, the matrix $R_{x}(m)$ has rank $M$, regardless of $L$, as long as $L \geq M$. Generally, the performance of the estimators is largely dependent on the accuracy of signal and noise subspace estimation from the correlation matrix of the noisy data $R_{y}(m)$. For large $L$, the columns of $W_{1}$ tend to be closer to the signal vectors $\mathbf{e}\left(f_{i}\right)$, and the smallest $L-M$ eigenvalues $\left\{\lambda_{i}\right\}_{i=M+1}^{L}$ tend to cluster around zero. This can be justified from the following observation. If $L$ is chosen such that $L=N$, then the matrix $(1 / \sqrt{N}) V$ is orthogonal, each column of which is an eigenvector of $R_{x}(m)$ with eigenvalue $\left|A_{i}\right|^{2}$. Thus, for sufficiently large $L$, we may expect that the eigenvalues and eigenvectors of $R_{x}(m)$ will be close to the signal vectors $\left\{\mathbf{e}\left(f_{i}\right)\right\}_{i=1}^{M}$. The effect of the parameter $L$ on the performance of MUSIC is studied in [11], where it is shown that a choice of $L$ such that $N / 3<L<N / 2$ would give reasonably good estimates.

To examine the effect of $M$, let $M_{1}$ be an estimate of the actual number of sinusoids such that $M_{1}>M$. Let $\bar{U}_{1}$ be a matrix of the most significant $M_{1}$ eigenvectors, and let $\bar{U}_{2}$ be a matrix of the least significant $L-M_{1}$ eigenvectors. Then, both $P_{2}(f)=\mathbf{e}^{*}(f) \bar{U}_{2}\left(\bar{U}_{2}^{*} \bar{U}_{2}\right)^{-1} \bar{U}_{2}^{*} \mathbf{e}(f)=0$ and $Q_{2}(f)=$ $\mathbf{e}^{*}(f) \bar{U}_{1}\left(\bar{U}_{1}^{*} \bar{U}_{1}\right)^{-1} \bar{U}_{1}^{*} \mathbf{e}(f)=L$ hold. While underestimating $M_{1}$ implies that $U_{2}$ has signal vectors, $P_{2}$ will display $r$ misestimated peaks, whereas $Q_{2}$ will display $M_{1}-r$ peaks. This suggests that to obtain an estimate of $M$, we can pick any $M$ and compute the zeros of both $P_{2}$ and $Q_{2}$. The number of peaks in both cases is $M$.

Using similar analysis to that of [6], we can show that for large $L$ and $M$, the accuracy may indeed improve significantly. The effect of $M$ on the MUSIC frequency estimator is studied in [10] and [11], where it is demonstrated that overestimation of $M$ leads to cleaner noise eigenvectors of $R_{y}(m)$. It is noted from simulations that this method is insensitive to the overestimation of $M$. In most applications, the number of sinusoids $M$ is not known a a priori. The number $M$ can be estimated using higher order lags by using the decomposition in ii) of Algorithm 2 above and examining the eigenvalues of the diagonal matrix $\Sigma$.

Remark 4.2-Estimation of the Amplitudes: Once the frequencies are estimated, the amplitudes $A_{1}, \cdots, A_{M}$ are then 
estimated by solving Vandermonde systems of equations.

$$
\begin{gathered}
{\left[\begin{array}{cccc}
1 & 1 & \cdots & 1 \\
e^{-2 \pi j f_{1}} & e^{-2 \pi j f_{2}} & \cdots & e^{-2 \pi j f_{M}} \\
\vdots & \vdots & \vdots & \vdots \\
e^{-2 \pi j(N-1) f_{1}} & e^{-2 \pi j(N-1) f_{2}} & \cdots & e^{2 \pi j(N-1) f_{M}}
\end{array}\right]} \\
\cdot\left[\begin{array}{c}
\left|A_{1}\right|^{2} \\
\left|A_{2}\right|^{2} \\
\vdots \\
\left|A_{M}\right|^{2}
\end{array}\right]=\left[\begin{array}{c}
r_{y}(0) \\
\vdots \\
r_{y}(N-1)
\end{array}\right]
\end{gathered}
$$

to obtain

$$
\left|A_{i}\right|^{2}=\frac{1}{N} \sum_{k=0}^{N-1} z_{i}^{* k} r_{y}(k), \quad i \in[1, M] .
$$

Here, $z_{i}=e^{-2 \pi j f_{i}}$. Note that this can not be applied to the raw data because of the presence of the phase terms.

\section{FREQUENCY ESTIMATION USING DATA DECIMATION}

Data decimation or downsampling has been considered before in the context of spectral estimation [19], [20]. The main advantage of this technique is that only part of the measured data is used, which results in a large reduction in the computations. However, the main disadvantage of using data decimation is that it works only if the frequencies occupy a relatively small region; otherwise, for a large decimation factor, aliasing will occur, leading to ambiguity in resolving frequencies. To alleviate this problem, the frequency range could be divided into sub-bands and filter out those outside the band of interest. However, this will not completely eliminate aliasing since it is very difficult to implement an ideal passband filter. In this section, a data decimation technique in conjunction with signal and/or noise subspace methods discussed earlier is developed. The decimation or downsampling of the input data sequence stretches the frequency scale by the decimation factor $d$, thus offering better isolation of the neighboring frequencies and thereby reducing the interference caused by the proximity of other frequency components.

To understand the effect of decimation, assume as before that the signal $y(k)=\sum_{i=1}^{M} A_{i} e^{-j\left(2 \pi k f_{i}-\phi_{i}\right)}+v(k)$ is decimated with decimation factor $d$ so that the decimated signal is $z(k)=y(k d)=\sum_{i=1}^{M} A_{i} e^{-j\left(2 \pi k d f_{i}-\phi_{i}\right)}+v(k d)$. Then $r_{z}(m)=E\left\{y(k d) y[(k-m) d]^{*}\right\}=r_{y}(m d)$. Therefore, we have (10), shown at the bottom of the page, and

$$
R_{z}(m)=R_{x}(m d)+R_{v}(m d) .
$$

As shown before, if $v(k)$ is a colored noise process modeled by an $s$ th-order ARMA process $v(k)=b_{1} v(k-1)+\cdots+$ $b_{r} v(k-r)+w(k)+a_{1} w(k-1)+\cdots+a_{s} w(k-s)$, where $w(k)$ is a white process, then if the poles of $v$ are inside the unit circle, we have $\lim _{m \rightarrow \infty} r_{v}(m)=0$, which leaves $r_{y}(m)$ as a sum of pure sinusoids for sufficiently large $m$. In the standard MUSIC algorithm, the matrix $R_{z}(0)$ is used to extract the noise and signal subspaces. If the correlation of the noise is an exponentially decaying sequence, it is expected that the disturbance of the noise on the correlation $R_{z}(0)$ will be less severe since the correlation matrix $R_{v}(0)$ will be close to a diagonal matrix, i.e., decimation plays a role of whitening the noise and thus improves the performance of the MUSIC algorithm. However, when $m>0$, the elements of $R_{v}(m)$ tend to be relatively small, and thus, $R_{z}(m)$ is a slightly perturbed version of $R_{x}(m)$.

As mentioned before, decimation may result in aliasing, particularly when the decimation factor is large. To alleviate the problem of aliasing, we can use this decimation process to estimate the $e^{-2 \pi j d f_{i}}$ 's and the $e^{-2 \pi j(d+1) f_{i}}$, s, which requires solving two problems. The main result concerning the retrieval of the $f_{i}$ 's using a decimation factor $d$ is given in the next theorem.

Theorem 5.1: Assume that $L>M$, and let $F_{d}$ and $F_{d+1}$ be the matrices that minimize the two problems

$$
\operatorname{Trace}\left\{\sum_{k}\left[\mathbf{y}_{k d}-F_{d \mathbf{y}_{(k-1) d}}\right]\left[\mathbf{y}_{k d}-F_{d \mathbf{y}_{(k-1) d}}\right]^{*}\right\}
$$

and

$$
\begin{gathered}
\operatorname{Trace}\left\{\sum_{k}\left[\mathbf{y}_{k(d+1)}-F_{d+1} \mathbf{y}_{(k-1)(d+1)}\right]\right. \\
\left.\left[\mathbf{y}_{k(d+1)}-F_{d+1} \mathbf{y}_{(k-1)(d+1)}\right]^{*}\right\}
\end{gathered}
$$

respectively. Then, in the noiseless case, $F_{d}$ is similar to $F^{d}$, and $F_{d+1}$ is similar to $F^{d+1}$, where $F$ is as defined in (7b).

Proof: The proof follows from Proposition 3.2 and (7a) and $(7 \mathrm{~b})$.

If $R_{y_{d}}(k)$, the correlation matrix for the decimated signal is of rank $M$; then, this problem has a unique minimizer obtained by setting

$$
\begin{aligned}
F_{d}= & \frac{1}{2}\left\{\sum_{k} R_{y_{d}}^{*}(k) R_{y_{d}}(k)\right\}^{-1} \\
& \cdot\left\{\sum_{k}\left[R_{y_{d}}^{*}(k) R_{y_{d}}(k+1)+R_{y_{d}}^{*}(k+1) R_{y_{d}}(k)\right\} .\right.
\end{aligned}
$$

$$
R_{z}(m)=E\left[\mathbf{y}_{\left.k d \mathbf{y}_{(k-m) d}^{*}\right]}\right]=\left[\begin{array}{cccc}
r_{y}(m d) & r_{y}[(m-1) d] & \cdots & r_{y}[(m-L+1) d] \\
r_{y}[(m+1) d] & r_{y}(m d) & \cdots & r_{y}[(m-L+2) d] \\
\vdots & \vdots & \vdots & \vdots \\
r_{y}[(m+L-1) d] & r_{y}[(m+L-2) d] & \cdots & r_{y}(m d)
\end{array}\right]
$$


However, since $M$ is not known for most applications, we solved the generalized eigenvalue problem

$$
\begin{aligned}
& 2\left\{\sum_{k} R_{y_{d}}^{*}(k) R_{y_{d}}(k)\right\} F \\
& \quad=\left\{\sum_{k}\left(R_{y_{d}}^{*}(k) R_{y_{d}}(k+1)+R_{y_{d}}^{*}(k+1) R_{y_{d}}(k)\right\} .\right.
\end{aligned}
$$

The solution of (11) can be obtained by an algorithm analogous to that in Algorithm 2.

The eigenvalues of $F_{d}$ and $F_{d+1}$ are $e^{-2 \pi j d f_{i}}$ and $e^{-2 \pi j(d+1) f_{i}}$, respectively, for $i \in[1, M]$. Unfortunately, these matrices are not simultaneously diagonalizable; thus, nonsingular matrices $Q_{d}$ and $Q_{d+1}$ have to be determined so that $Q_{d}^{-1} F_{d} Q_{d}=\operatorname{diag}\left(e^{-2 \pi j d f_{i}}\right)$, and $Q_{d+1}^{-1} F_{d+1} Q_{d+1}=$ $\operatorname{diag}\left(e^{-2 \pi j(d+1) f_{i}}\right)$. We can employ all the sub-bands of downsampling (which are sometimes referred to as polyphase components) to obtain more reliable estimates of the $f_{i}$, i.e., by considering all the subsampled signals $y_{i}(k)=y(k d+i-1)$, $i=1, \cdots, d$ and computing

$$
\bar{R}_{z}(m)=\sum_{i=1}^{d} R_{y_{i}}(d m)
$$

where

$$
R_{y_{i}}(d m)=E\left[\mathbf{y}_{d k+i-1} \mathbf{y}_{d(k-m)+i-1}^{*}\right] .
$$

In the next algorithm, we incorporate the decimation process with Algorithms 1 or 2.

Algorithm 3:

i) To apply decimation for resolution improvement, choose the decimation factors $d, d+1$, and consider the new signal $z(k)=y(k d)=\sum_{i=1}^{M} A_{i} e^{-j\left(2 \pi k d f_{i}-\phi_{i}\right)}+$ $v(k d)$.

ii) Compute the covariance matrix $R_{z}(m)=$ $E\left[\mathbf{y}_{k \cdot d} \mathbf{y}_{(k-m) d}^{*}\right]$.

iii) Apply Algorithms 1 or 2 to estimate the frequencies $f_{i}^{(d)}$ and $f_{i}^{(d+1)}$.

iv) Compute $u_{i}=\left[f_{i}^{(d)}+k_{1}\right] / d$ and $v_{i}=\left[f_{i}^{(d+1)}+\right.$ $\left.k_{2}\right] / d+1$ for $i=1, \cdots, M$ and $k_{1}=0, \cdots, d-1$, $k_{2}=0, \cdots, d$.

v) Choose the set with the best matching between $u_{i}$ 's and $v_{j}$ 's, i.e., $\left|u_{i}-v_{j_{i}}\right|=\min _{j}\left\{\left|u_{i}-v_{j}\right|\right\}$. Then, the frequencies can be estimated as $f_{i}=\left(u_{i}+v_{j_{i}}\right) / 2$.

vi) Compute the amplitudes $A_{i}$ as in Remark 4.2.

Remark 5.1: To examine the effect of decimation on the sensitivity of parameters, consider a decimation factor $d$. Assuming the noise is modeled by an AR model with poles $w_{j}, j=1, \cdots, K$, the polynomial that governs the poles of this problem before decimation is

$$
\prod_{i=1}^{M}\left(z-z_{i}\right) \prod_{j=1}^{K}\left(z-w_{j}\right)
$$

where the $z_{i}$ represents the poles of the signal, and the $w_{j}$ represents the poles of noise. It will be assumed, as in most applications, that the poles of the noise are strictly inside
TABLE I

Various Frequency Estimators. Data: Two Complex SinUSOIDS AT FREQUENCIES 0.2 AND 0.6 IN COLORED

NoISE. SNR $=5,0,-5,-10 \mathrm{~dB}$, Dimension of Data Vectors $L=30$, Dimension of Signal Subspace $M=2$

\begin{tabular}{c|c|c|c|c|c}
\hline Lagged Matrix & $\mathrm{SNR}$ & $f_{1}$ & $\sigma_{f_{1}}$ & $f_{2}$ & $\sigma_{f_{2}}$ \\
\hline$R_{y}(1)$ & $5 \mathrm{~dB}$ & 0.2144 & 0.0008066 & 0.6076 & 0.0006751 \\
\hline$R_{y}(1)$ & $0 \mathrm{~dB}$ & 0.2145 & 0.0027 & 0.6079 & 0.0014 \\
\hline$R_{y}(1)$ & $-5 \mathrm{~dB}$ & 0.2129 & 0.0398 & 0.6102 & 0.0120 \\
\hline$R_{y}(1)$ & $-10 \mathrm{~dB}$ & 0.2354 & 0.10330 & 0.6262 & 0.0800 \\
\hline \hline$R_{y}(2)$ & $5 \mathrm{~dB}$ & 0.1941 & 0.0008825 & 0.6148 & 0.0008912 \\
\hline$R_{y}(2)$ & $0 \mathrm{~dB}$ & 0.1947 & 0.0018 & 0.6146 & 0.0017 \\
\hline$R_{y}(2)$ & $-5 \mathrm{~dB}$ & 0.2137 & 0.0731 & 0.6141 & 0.0398 \\
\hline$R_{y}(2)$ & $-10 \mathrm{~dB}$ & 0.2065 & 0.1060 & 0.6039 & 0.1211 \\
\hline$R_{y}(3)$ & $5 \mathrm{~dB}$ & 0.2078 & 0.0008829 & 0.5873 & 0.0008161 \\
\hline$R_{y}(3)$ & $0 \mathrm{~dB}$ & 0.2123 & 0.0281 & 0.5873 & 0.0016 \\
\hline$R_{y}(3)$ & $-5 \mathrm{~dB}$ & 0.2191 & 0.0587 & 0.5977 & 0.0400 \\
\hline$R_{y}(3)$ & $-10 \mathrm{~dB}$ & 0.2425 & 0.0999 & 0.6153 & 0.1117 \\
\hline \hline$R_{y}(1)+R_{y}(3)$ & $5 \mathrm{~dB}$ & 0.2036 & 0.0007355 & 0.5983 & 0.0005541 \\
\hline$R_{y}(1)+R_{y}(3)$ & $0 \mathrm{~dB}$ & 0.2031 & 0.0014 & 0.5984 & 0.0017 \\
\hline$R_{y}(1)+R_{y}(3)$ & $-5 \mathrm{~dB}$ & 0.2147 & 0.0451 & 0.6046 & 0.0544 \\
\hline$R_{y}(1)+R_{y}(3)$ & $-10 \mathrm{~dB}$ & 0.2056 & 0.1032 & 0.5990 & 0.1103 \\
\hline
\end{tabular}

the unit circle. After the noisy signal is decimated, the new characteristic polynomial becomes

$$
\prod_{i=1}^{M}\left(z-z_{i}^{d}\right) \prod_{j=1}^{K}\left(z-w_{j}^{d}\right) .
$$

This shows that the poles corresponding to the noiseless signal are $z_{i}^{d}$, which still lie on the unit circle, whereas those corresponding to the noise are $w_{j}^{d}$. Since $w_{i}$ 's lie inside the unit circle, their powers get smaller in magnitude as $d$ gets larger. This demonstrates that the effect of noise diminishes as the decimation factor $d$ becomes large. Thus, decimation, together with correlations of higher order lags, will have the advantage of significantly reducing the effects of noise. However, this entails the solution of two optimization problems, which is offset by the reduced number of computations associated with the decimation process.

\section{EXPERIMENTAL RESULTS}

In this section, we illustrate the performance of some of the algorithms developed in this paper on two different examples.

Example 1: Consider the data sequence generated by the equation

$$
y(k)=A_{1} e^{j 2 \pi f_{1} k}+A_{2} e^{j 2 \pi f_{2} k}+v(k)
$$

where $f_{1}=0.2$ and $f_{2}=0.6$, and $k=1,2, \cdots, 60$. The amplitudes of the sinusoids were $A_{1}=1.0$ and $A_{2}=1.0$. The SNR for either sinusoids is defined as $10 \log _{10}\left(\sigma_{x}^{2} / \sigma_{v}^{2}\right)$, where $x(k)=A_{1} e^{j 2 \pi f_{1} k}+A_{2} e^{j 2 \pi f_{2} k}$ and $\sigma_{x}^{2}, \sigma_{v}^{2}$ are the variances of signal $x(k)$ and colored noise $v(k)$, respectively. The lagged correlation matrices are constructed using forwardbackward method to increase the robustness. The size of each matrix is chosen to be $(L=30)$, which in the absence of noise has effective rank two. The QZ routine on MATLAB is employed for the computation of the generalized eigenvectors and eigenvalues required to implement Algorithm 1. The mean values and standard deviations of the estimated frequencies are given in Table I for a set of 40 random experiments 

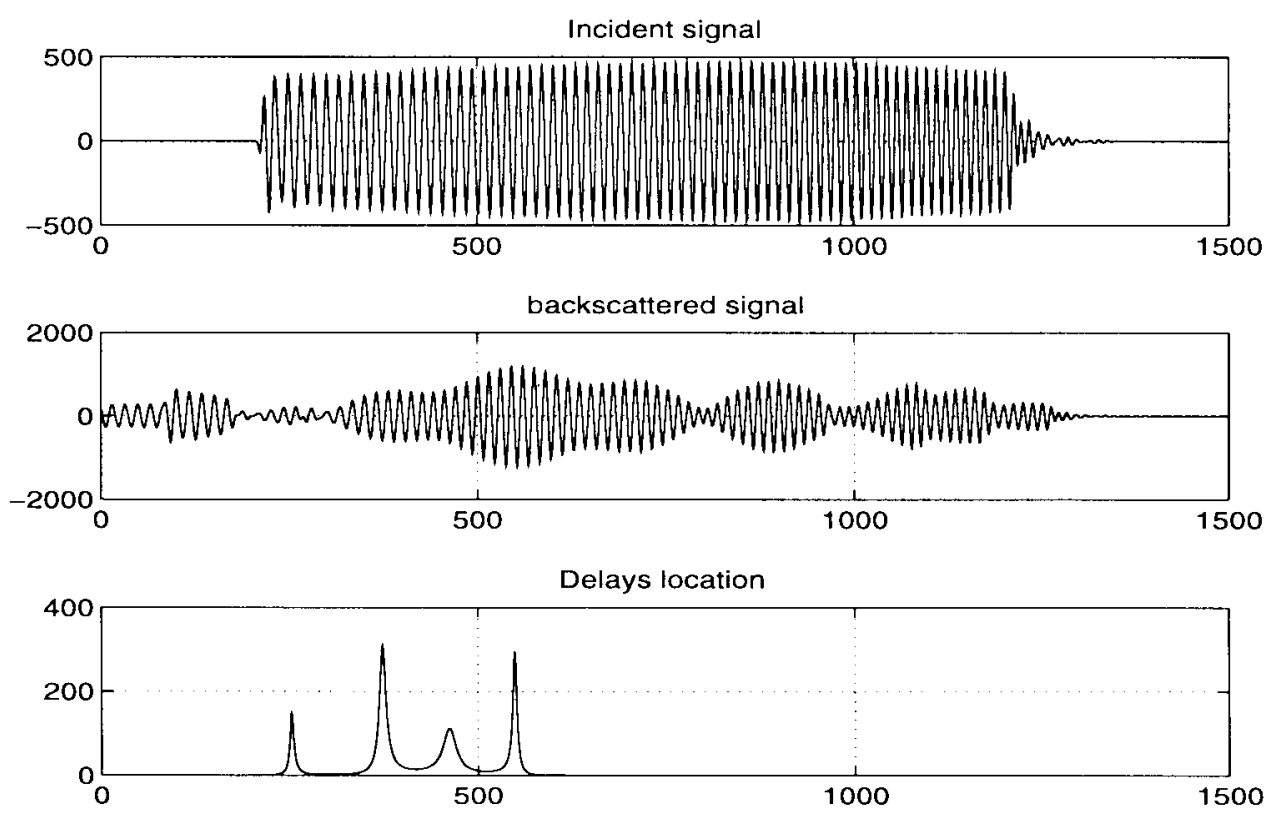

Fig. 1. Incident and simulated backscattered for Case 1 with spectral peaks at delays $=249,369,456,548$.
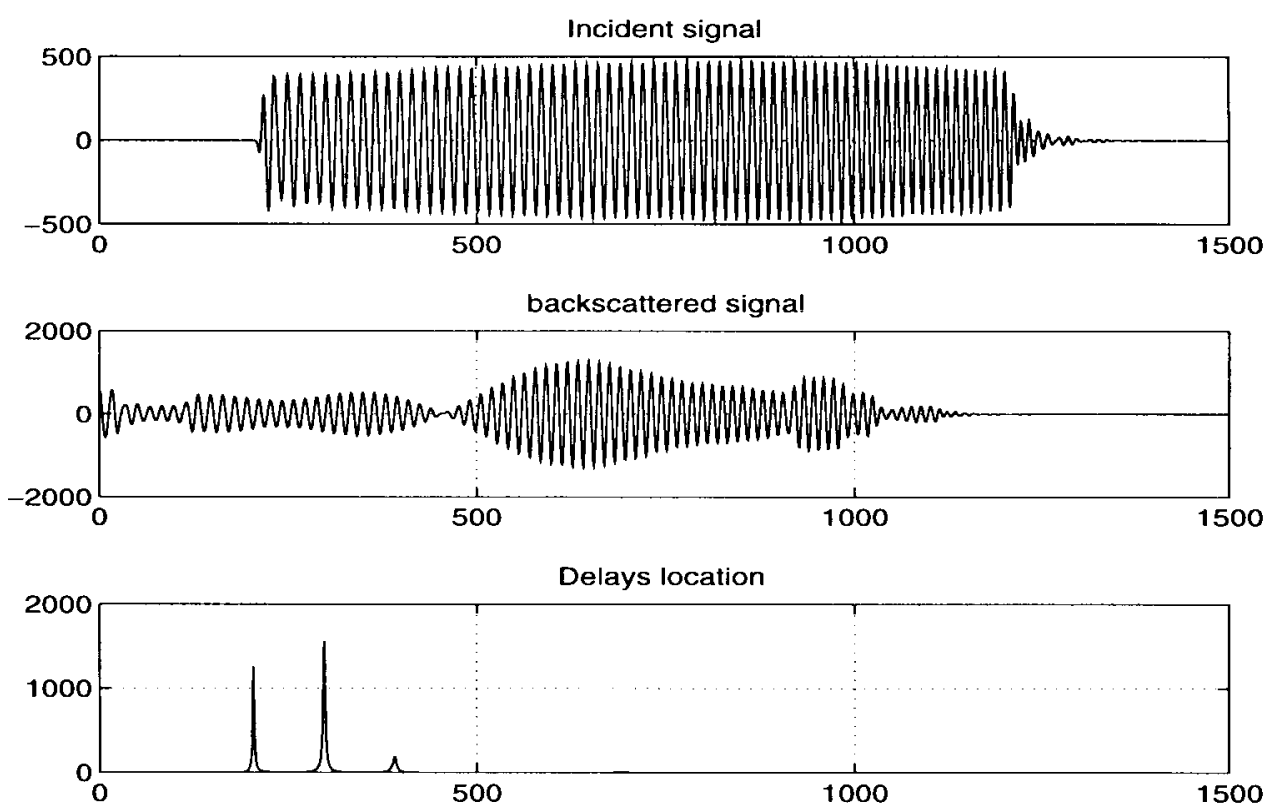

Fig. 2. Incident and simulated backscattered for Case 2 with spectral peaks at delays $=201,295,389$.

for different SNR (SNR $=5,0,-5,-10 \mathrm{~dB}$ ) and different lagged correlations $\left\{R_{y}(m)\right\}_{m=1}^{3}$. The dimension of the signal subspace is considered to be $M=2$, using the correlation matrix $R_{y}(0)$. It is noticed that the performances in these cases are close to those obtained using the correlation matrix $R_{y}(0)$.

The last four rows consider the case where combination of lagged correlation matrices, namely, $R_{y}(1)+R_{y}(3)$ is used. The results show some improvements over those using $R_{y}(1)$, $R_{y}(2)$, or $R_{y}(3)$ alone. It is also noted that false peaks will appear if $M>4$.

Example 2: Next, we consider the time-delay estimation problem and test the performance of Algorithm 2 described in Section IV. We examined several data sets consisting of an incident and simulated backscattered signals. The incident signal was a wideband linear FM, and the simulated backscattered return was generated using (1) and (2) with $v(k)=\sum_{i=1}^{2} b_{i} w(k-i)$ and $w(k)$, which is a white noise with zero mean and unit variance. The coefficients $b_{1}$ and $b_{2}$ were taken to be $b_{1}=0.7071$ and $b_{2}=0.7071$ so that $b_{1}^{2}+b_{2}^{2}=1$. The purpose of this study was to analyze the performance of Algorithm 2 under various conditions such as low amplitude and closely spaced specular components in the simulated backscattered signals. Three simulated backscattered signals were generated with the actual time-delays shown in Table II. In all cases, the FM incident signal was of length 4096 samples. These cases contained four components with nonuniform separations.

In the first case, there were four delays, as shown in Table I, with amplitudes $A_{1}=0.7, A_{2}=1.0, A_{3}=0.8$, and $A_{4}=0.8$ with zero phase. When data vector length $L=40, M=10$, 

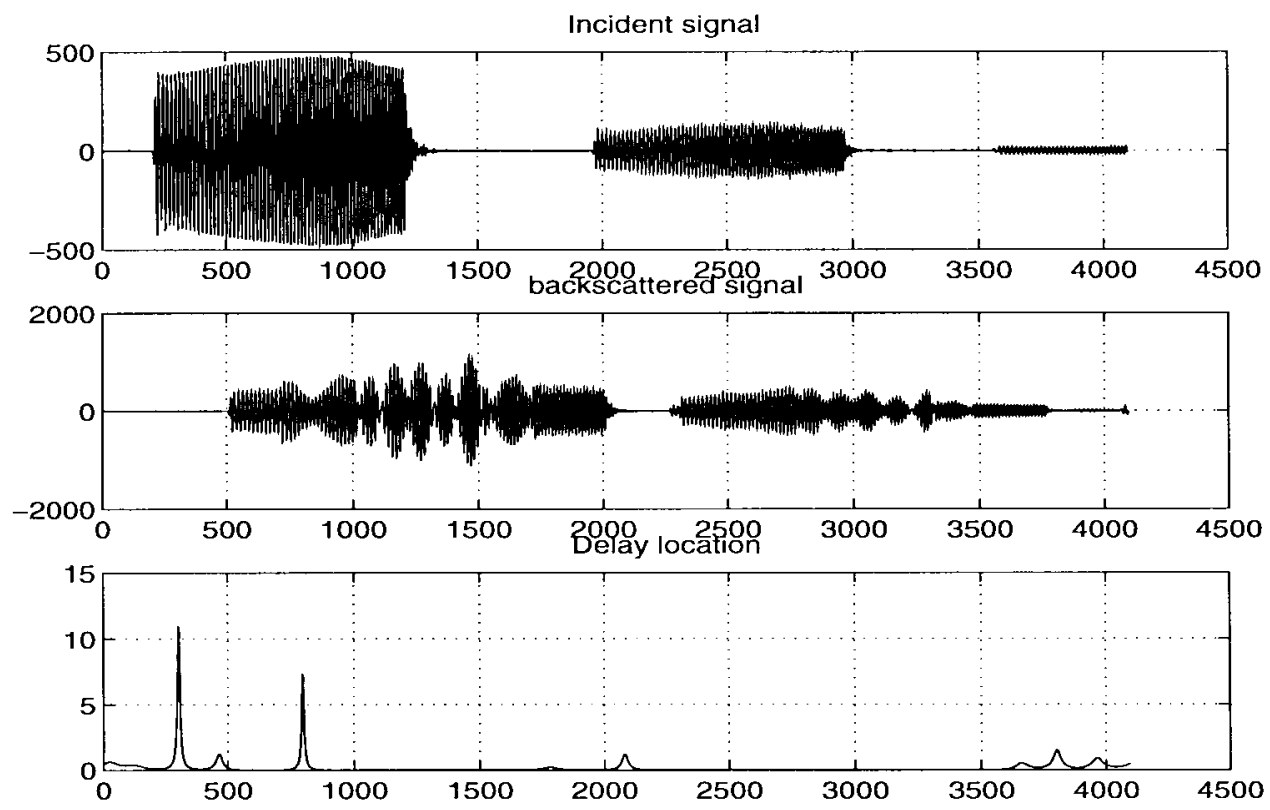

Fig. 3. Incident and simulated backscattered for Case 3 with spectral peak at delay $=303,465,797,2082$.
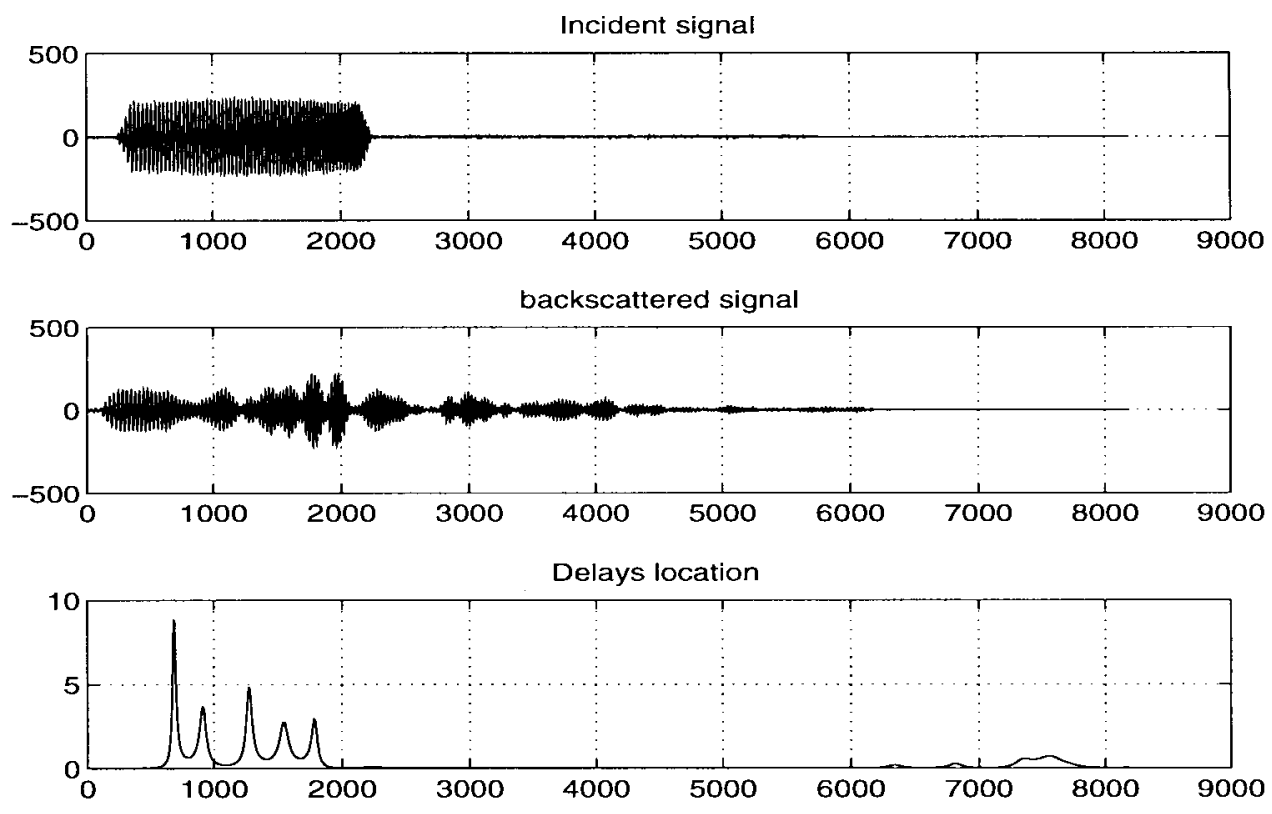

Fig. 4. Incident and real backscattered for Case 4 with main spectral peak at delay $=650$.

and $\mathrm{SNR}=30 \mathrm{~dB}$ were used, the result of Algorithm 2 is shown in Fig. 1. This result shows that if the separation between delays is sufficiently large, we can recover the delays very accurately within four samples in this case.

In the second case, we also have four delays as shown in Table I with amplitudes $A_{1}=1.0, A_{2}=0.7, A_{3}=0.9$, and $A_{4}=0.5$. When $L=40, M=10$, and $\mathrm{SNR}=30 \mathrm{~dB}$, the results of Algorithm 2 are shown in Fig. 2. Clearly, one of the delays is missing due to closely spaced second and third delays. The fourth delay is detected, although its amplitude was smaller than all the others. This is due to the fact that the fourth time delay is well separated from the others.

In the third case, the amplitudes of the components were $A_{1}=1, A_{2}=0.5, A_{3}=1$, and $A_{4}=0.6$. The results are shown in Fig. 3 for when $L=40, M=10$, and SNR $=40$ $\mathrm{dB}$. As can be seen, all the delays are accurately estimated: even those with small amplitudes.

The fourth experiment involved a real backscattered signal of length 8192. The data was obtained from a submerged elastic target that had the form of a tapered notched cylinder with flattened ends and rivets and an aspect ratio of 4 to 1 . The same linear FM incident signal with a time-bandwidth product of $T B=20$ was used. The signal was set to sweep over the midfrequency band [1]. The returns from each object were collected over $360^{\circ}$ in $5^{\circ}$ increments to produce 72 data records of differing aspect angle per object. Note that $0^{\circ}$ corresponds to broadside incident. The measurements were performed under controlled operating and environmental 
TABLE II

Actual Delays versus Estimated Ones for Three Cases

\begin{tabular}{c|c|c}
\hline$\overline{\text { Cases }}$ & Actual Delays & Estimated Values \\
\hline 1 & $250,370,460,550$ & $249,369,456,548$ \\
\hline 2 & $200,270,310,400$ & $201,295,389$ \\
\hline 3 & $300,500,800,2100$ & $303,465,797,2082$ \\
\hline
\end{tabular}

conditions. It was observed in the results that depending on the angle of incidence, the number of specular reflections varies. For the broadside incidence, the only prominent component was detected around $\Delta=650$. Fig. 4 shows the results for this case. The above results indicate the effectiveness of the proposed schemes in detecting multiple specular returns and estimating their associated delays.

\section{CONCLUSION}

Correlations with lags were used to develop new approaches for the estimation of the time delays associated with multiple specular components in the acoustic backscattered signal. The signal parameters were estimated by using MUSIClike and pencil-based methods. It was demonstrated through simulations that when the additive noise is colored with unknown autocorrelation function, these algorithms are particularly effective in estimating the time delays (or frequencies). The main advantage of the estimators proposed in this paper is that using correlations of higher lags significantly reduces the effects of additive noise, hence leading to more robust estimators. Decimation was shown to improve this robustness property. Simulation results on the two examples clearly demonstrated the effectiveness of the proposed schemes.

\section{APPENDIX A}

PROOF OF PROPOSITION 3.2

Equation (6) follows from Proposition 3.1. It can easily be verified that $R_{x}(m)=V D S^{m} D^{*} V^{*}$, where

$$
V=\left[\begin{array}{cccc}
1 & 1 & \cdots & 1 \\
e^{-2 \pi j f_{1}} & e^{-2 \pi j f_{2}} & \cdots & e^{-2 \pi j f_{M}} \\
\vdots & \vdots & \vdots & \vdots \\
e^{-2 \pi j(L-1) f_{1}} & e^{-2 \pi j(L-1) f_{2}} & \cdots & e^{2 \pi j(L-1) f_{M}}
\end{array}\right]
$$

$D=\operatorname{diag}\left(A_{1}, A_{2}, \cdots, A_{M}\right)$, and $S=\operatorname{diag}\left(e^{-2 \pi j f_{1}}\right.$, $\left.e^{-2 \pi j f_{2}}, \cdots, e^{-2 \pi j f_{M}}\right)$. This decomposition property of $R_{x}(m)$ is used to prove i)-vi). Clearly, we have $F V=V S$ and $S S^{*}=I$, which give $R_{x}(m)=V D S^{m} D^{*} V^{*}=$ $V S D S^{m} D^{*} S^{*} V^{*}=F V D S^{m} D^{*} V^{*} F^{*}=F R_{x}(m) F^{*}$. Similarly, $R_{x}(m+1)=V D S^{m+1} D^{*} V^{*}=V S D S^{m} D^{*} V^{*}=$ $F V D S^{m} D^{*} V^{*}=F R_{x}(m)$. Analogously, we can show that $R_{x}(m)=R_{x}(m+1) F^{*}$. Items ii), iv), and v) in Proposition 3.2 follow from iterating i) and iii). To prove vi), we have that the matrix $R_{x}(m)$ is of rank $M$ when $L>M$ since the principal $M \times M$ submatrix of $R_{x}(m)$ is nonsingular with determinant $\prod_{i=1}^{M}\left|A_{i}\right|^{2} e^{-2 \pi j f_{i}}|V|^{2}\left(e^{-2 \pi j f_{1}}, \cdots, e^{-2 \pi j f_{M}}\right)$, where $|V|$ is the determinant of the matrix $V$. The last conclusion can also be seen from the factorization

$$
R_{x}(m)=P^{*} S^{m} P
$$

where $P:=V D$, i.e.,

$$
P=\left[\begin{array}{cccc}
A_{1} & A_{1} e^{2 \pi j f_{1}} & \cdots & A_{1} e^{2 \pi j(L-1) f_{1}} \\
A_{2} & A_{2} e^{2 \pi j f_{2}} & \cdots & A_{2} e^{2 \pi j(L-1) f_{2}} \\
\vdots & \vdots & \vdots & \vdots \\
A_{M} & A_{M} e^{2 \pi j f_{M}} & \cdots & A_{M} e^{2 \pi j(L-1) f_{M}}
\end{array}\right]
$$

which is clearly of rank $M$, provided $A_{i} \neq 0$ for $i \in[1, M]$.

APPENDIX B

ProOF of Proposition 3.3

Let $G=\left[\begin{array}{llll}\mathbf{g}_{M+1} & \mathbf{g}_{M+2} & \cdots & \mathbf{g}_{L}\end{array}\right]$; then, $G$ is of rank $L-M, F^{*} \mathbf{g}_{i}=\mathbf{g}_{i+1}$, for $i=M+1, M+2, \cdots, L-1$ and $F^{*} \mathbf{g}_{L}=0$. The last equation means that the null space of $F^{*}$ is contained in the null space of $R_{x}(m)$. It can easily be noticed that $F^{* L-M} \mathbf{g}_{i}=0, i=M+1, \cdots, L$. Therefore, $\mathcal{N}\left(F^{* L-M}\right) \subset \mathcal{N}\left[R_{x}(m)\right]$. From Proposition 3.2, $R_{x}(m)$ is of rank $M$, and hence, $\mathcal{N}\left[R_{x}(m)\right]$ is $(L-M)$ dimensional. It follows that $\mathcal{N}\left(F^{* L-M}\right)=\mathcal{N}\left[R_{x}(m)\right]$. To show that $\mathcal{N}\left[R_{x}^{*}(m)\right]=\mathcal{N}\left[R_{x}(m)\right]$, we have $R_{x}^{*}(m)=$ $\left[F^{L-M} R_{x}(m+L-M)\right]^{*}=R_{x}^{*}(m-L+M) F^{* L-M}$. This gives $\mathcal{N}\left(F^{* L-M}\right) \subset \mathcal{N}\left[R_{x}^{*}(m)\right]$. Since rank $R_{x}(m)$ and $R_{x}^{*}(m)$ are the same, and each equals $M$, and the conclusion follows.

\section{REFERENCES}

[1] M. R. Azimi-Sadjadi, J. Wilbur, and G. Dobeck, "Isolation of resonance in acoustic backscatter from elastic targets using adaptive estimation schemes," IEEE J. Oceanic Eng., vol. 20, pp. 346-353, Oct. 1995.

[2] M. A. Hasan and M. R. Azimi-Sadjadi, "A modified block FTF adaptive algorithm with applications to underwater target detection," IEEE Trans. Signal Processing, vol. 44, pp. 2172-2185, Sept. 1996.

[3] W. S. Burdic, Underwater Acoustic System Analysis. Englewood Cliffs, NJ: Prentice-Hall, 1984.

[4] S. M. Kay, Modern Spectral Estimation, Theory and Applications. Englewood Cliffs, NJ: Prentice-Hall, 1988.

[5] D. W. Tufts and R. Kumaresan, "Estimation of frequencies of multiple sinusoids; Making linear prediction perform like maximum likelihood," Proc. IEEE, vol. 70, pp. 975-989, Sept. 1982.

[6] J. T. Karhunen and J. Joutsenalo, "Sinusoidal frequency estimation by signal subspace approximation," IEEE Trans. Acoust., Speech, Signal Processing, vol. 40, pp. 2961-2972, Dec. 1992.

[7] D. B. Rao and K. V. S. Hari, "Performance analysis of root-music," IEEE Trans. Acoust., Speech, Signal Processing, vol. 37, pp. 1939-1949, Dec. 1989.

[8] R. H. Roy and T. Kailath, "ESPRIT-estimation of signal parameters via rotational invariance techniques," IEEE Trans. Acoust., Speech, Signal Processing, vol. 37, pp. 984-995, July 1989.

[9] Y. Hua and T. K. Sarkar, "On SVD for estimating generalized eigenvalues of singular matrix pencils in noise," IEEE Trans. Signal Processing, vol. 39, pp. 892-899, Apr. 1991.

[10] P. Stoica, T. Soderstrom, and F. Ti, "Asymptotic properties of the highorder Yule-Walker estimates of sinusoidal frequencies," IEEE Trans. Signal Processing, vol. 37, pp. 1721-1734, Nov. 1989.

[11] P. Stoica and T. Soderstrom, "Statistical analysis of MUSIC and subspace rotation estimates of sinusoidal frequencies," IEEE Trans. Signal Processing, vol. 39, pp. 1836-1847, Aug. 1991.

[12] S. M. Kay and A. K. Shaw, "Frequency estimation by principal component AR spectral estimation method without eigendecomposition," IEEE Trans. Acoust., Speech, Signal Processing, vol. 36, pp. 95-101, Jan. 1988.

[13] D. Tufts and C. D. Melissinos, "Simple effective computation of principal eigenvectors and their eigenvalues and application to high-resolution of frequencies," IEEE Trans. Acoust., Speech, Signal Processing, vol. ASSP-34, pp. 1046-1052, Oct. 1986.

[14] V. T. Ermolaev and A. B. Gershman, "Fast algorithm for minimumnorm direction-of-arrival estimation," IEEE Trans. Signal Processing, vol. 42, pp. 2389-2394, Sept. 1994. 
[15] V. Negesha and S. Kay, "Maximum likelihood estimation for array processing in colored noise," IEEE Trans. Signal Processing, vol. 44, pp. 169-180, Feb. 1996.

[16] B. Friedlander and J. Frances, "On the accuracy of estimating the parameters of a regular stationary process," IEEE Trans. Inform. Theory, vol. 42, pp. 1202-1211, July 1996.

[17] B. S. Garbow, J. M. Boyle, J. J. Dongarra, and C. B. Moler, Matrix Eigensystem Routines-EISPACK Guide Extension, G. Goos and J. Hortmanis, Eds. New York: Springer-Verlag, 1977.

[18] G. H. Golub and C. G. Van Loan, Matrix Computations, 2nd ed. Baltimore, MD: John Hopkins Univ. Press, 1989.

[19] W. M. Steedly, C. J. Ying, and R. L. Moses, "A modified TLS-prony method using data decimation," IEEE Trans. Signal Processing, vol. 42, pp. 2292-2203, Sept. 1994.

[20] M. J. Villalba and B. K. Walker, "Spectrum manipulation for improved resolution," IEEE Trans. Signal Processing, vol. 37, pp. 820-831, June 1989.

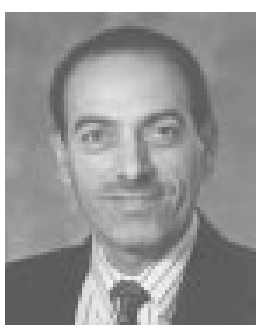

Mohammed A. Hasan received the Ph.D. degree in mathematics and the Ph.D. degree in electrical engineering from Colorado State University, Fort Collins, in 1991, and 1997, respectively.

$\mathrm{He}$ is currently with the Department of Electrical and Computer Engineering, University of Minnesota, Duluth. His research interests include adaptive systems, signal/image processing, estimation theory, control theory, numerical analysis, optimization, numerical linear algebra, and computational and applied mathematics.

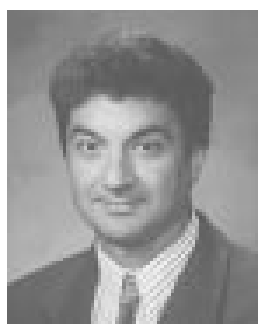

Mahmood R. Azimi-Sadjadi (SM'89) received the B.S. degree from the University of Tehran, Tehran, Iran, in 1977, the M.Sc. and Ph.D. degrees from the Imperial College, University of London, London, U.K., in 1978 and 1982, respectively, all in electrical engineering.

He served as an Assistant Professor in the Department of Electrical and Computer Engineering, University of Michigan, Dearborn. Since July 1986, he has been with the Department of Electrical Engineering, Colorado State University, Fort Collins, where he is now a Professor. He is also the Director of the Multisensory Computing Laboratory (MUSCL) at Colorado State. His areas of interest are digital signal/image processing, target detection and tracking, multidimensional system theory and analysis, adaptive filtering, system identification, and neural networks. His contributions in these areas have resulted in more than 100 journal and refereed conference publications. He is co-author of the book Digital Filtering in One and Two Dimensions (New York: Plenum, 1989).

Dr. Azimi-Sadjadi was the recipient of the 1993 ASEE-Navy Senior Faculty Fellowship Award, the 1991 CSU Dean's Council Award, the 1990 Battelle Summer Faculty Fellowship Award, and the 1984 Dow Chemical Outstanding Young Faculty Award of the American Society for Engineering Education. He is an Associate Editor of the IEEE Transactions on Signal Processing.

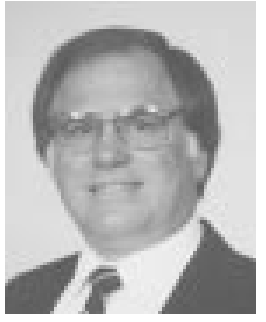

Gerald J. Dobeck received the B.S. degree in physics from the University of Massachusetts, Amherst, in 1970 and the M.S. and Ph.D. degrees in electrical engineering from the University of South Florida, Tampa, in 1973 and 1976, respectively.

Since 1976, he has been employed at the Coastal Systems Station, Naval Surface Warfare Center, Dahlgren Division, Panama City, FL. His curren research interests include automatic detection and classification of underwater targets in cluttered environments from synthetic/real aperture sonar imagery, the echo structure of acoustic sonar returns, underwater electrooptic imagery, and gradiometer/magnetometer signals. He is project leader of the Sensor Signal and Image Processing project under the Office of Naval Research 6.2 Mine Countermeasures program. In this, he is Technical Leader on the development of automated mine detection and classification algorithms for sonar imagery, magnetic gradiometer, and acoustic backscatter. He has authored or co-authored more than 60 technical reports and papers.

Dr. Dobeck received the 1981 and 1996 Commanding Officer/Technical Director Science and Technology Award. He is a reviewer for IEEE, ASME, and the Journal of Underwater Acoustics and has been session chair at past IEEE and SPIE conferences. 